\title{
Crossed-Derivative Based Sensitivity Measures for Interaction Screening
}

\author{
O. Roustant ${ }^{\mathrm{a}, *}$, J. Fruth $^{\mathrm{b}}$, B. Iooss ${ }^{\mathrm{c}}$, S. Kuhnt ${ }^{\mathrm{b}, \mathrm{d}}$ \\ ${ }^{a}$ Ecole Nat. Sup. des Mines, FAYOL-EMSE, LSTI, F-42023 Saint-Etienne, France \\ ${ }^{b}$ Faculty of Statistics, TU Dortmund University, Vogelpothsweg 87, 44227 Dortmund, \\ Germany \\ ${ }^{c}$ Electricité de France REDD, 6 quai Watier, F-78401, France \\ ${ }^{d}$ Department of Computer Science, Dortmund University of Applied Sciences and Arts, \\ Emil-Figge-Strasse 42, 44227 Dortmund, Germany
}

\begin{abstract}
Global sensitivity analysis is used to quantify the influence of input variables on a numerical model output. Sobol' indices are now classical sensitivity measures. However their estimation requires a large number of model evaluations, especially when interaction effects are of interest. Derivative-based global sensitivity measures (DGSM) have recently shown their efficiency for the identification of non-influential inputs. In this paper, we define crossed DGSM, based on second-order derivatives of model output. By using a $L^{2}$ Poincaré inequality, we provide a crossed-DGSM based maximal bound for the superset importance (i.e. total Sobol' indices of an interaction between two inputs). In order to apply this result, we discuss how to estimate the Poincaré constant for various probability distributions. Several analytical and numerical tests show the performance of the bound and allow to develop a generic strategy for interaction screening.
\end{abstract}

Keywords: Sensitivity analysis, Derivative-based sensitivity measure, Sobol decomposition, Interactions, Superset importance, Additive structure

\footnotetext{
*Corresponding author: roustant@emse.fr, Phone: +33 477426675, Fax: +33 477426666

Email addresses: fruth@statistik.tu-dortmund.de (J. Fruth), bertrand.iooss@edf.fr (B. Iooss), sonja.kuhnt@fh-dortmund.de (S. Kuhnt)
} 


\section{Introduction}

In engineering studies, computer models simulating physical phenomena and industrial systems often take as inputs a high number of numerical and physical variables. For the development, the analysis and the uses of such computer models, the global sensitivity analysis methodology is an invaluable tool [22]. Among quantitative methods, variance-based methods are used most often [23]. The main idea of these methods is to evaluate, by the way of so-called Sobol indices [24], how the variation of an input or a group of inputs contributes to the model output variability. Sobol indices of first order measure the effect of individual inputs, while second-order Sobol indices correspond to the influence of the interaction between two inputs (by excluding their individual effects). Moreover, the powerful total Sobol sensitivity index has been introduced to express the overall contribution of one specified input, including the effects of its interactions (second order, third order, ...) with all the other inputs [11]. More generally, the so-called superset importance of an input set $I$ has been defined in [17] and [12] as the sum of all Sobol' indices relative to the supersets of $I$. In the particular case of two inputs, the superset importance has been referred to as the total interaction index ([8]). When its value is zero, it means that there is no interaction term containing simultaneously the two inputs. By analogy with screening, this can be viewed as interaction screening.

However, obtaining all these Sobol sensitivity indices is rather costly in terms of the number of necessary model evaluations [23]. This seriously limits their use because industrial computer codes often require several minutes or hours to perform one run. Moreover, for large dimension (number of inputs larger than several tens), high-order Sobol' indices cannot be obtained by non-costly alternative methods based on metamodels or smoothing techniques $[29,18]$. Introduced by [26], an alternative global sensitivity estimator consists, for each input, in integrating the square derivative of the model output over the domain of the inputs. Called later the Derivative-based Global Sensitivity Measures (DGSM), they have been proved to be computationally more tractable than variance-based measures [15], allowing to manage the problem of large numbers of inputs. DGSM have only been finely studied and applied recently $[27,21,13]$. Moreover, these indices need the computation of the gradient of the model output with respect to the model inputs, which can be at least estimated by a finite-differences technique. However, if the computer model proposes the adjoint code to compute output derivatives 
[5], DGSM computations will be independent of the number of input parameters and sensitivity analysis can then be performed for models including several hundreds of inputs. Automatic differentiation tools [9] can be helpful to that purpose.

Recently, some authors have discovered links between DGSM and variancebased sensitivity indices. For an input following a uniform or normal probability distribution, [27] has proved that the total Sobol' index is bounded by a term involving a constant and the DGSM. [16] has developed a generalization of this inequality for a large class of continuous probability measures. Thus, it has been proved that the DGSM can be used for robust variable screening. In engineering applications, this inequality allows to develop a generic strategy to obtain global sensitivity information from DGSM and first-order Sobol indices [13].

In this paper we extend this approach to interactions, by proving a generalization of the inequality in the same large class of probability measures. We also obtain results for the class of log-concave measures. Thus, the secondorder derivatives contained in the Hessian of the model output are useful to investigate interactions. Such a link was investigated for instance by [7] in the context of statistical learning, but the connection to superset indices gives an original interpretation of it. While it is rare in practice that second-order derivatives of the model output are directly available, they can be computed by second-order finite differences or by automatic differentiation tools. In the same vein of [16], we also investigate by numerical tests the utility of the inequality in ranking the most influential interactions. While this ranking may be useful at first sight for superset importances more than for secondorder interactions, we can argue that in practice it is very often the case that second-order interactions are the only active ones.

In [28], a similar problem has been considered by using groups of inputs. The same kind of inequality links the total Sobol index of a group with the corresponding DGSM (equal to the integral of the square sum of the partial derivatives of each group input). If this latter measure is zero, it means that there is no interaction between the inputs inside the group. However, if the DGSM of a group is non zero, the total interaction index between two inputs can be zero, which is detected by the crossed DGSM.

The paper is organized as follows: Section 2 recalls some useful definitions of sensitivity indices (Sobol indices, superset importances, DGSM) and introduces the crossed DGSM. Section 3 establishes an inequality between superset importance and crossed DGSM. Section 4 focuses on the determi- 
nation of the Poincaré constant, which is used in the previous inequality. Section 5 develops the link between superset importance and crossed DGSM on classical analytical functions, while section 6 applies the inequality on numerical simulations on two test models. It illustrates how crossed DGSM can be used in practice. We conclude in Section 7.

\section{Global sensitivity measures}

\subsection{Variance-based sensitivity measures}

We consider a model output $Y=g(\mathbf{X})$ where $\mathbf{X}=\left(X_{1}, \ldots, X_{d}\right)$ is a vector of independent random variables with distribution $\mu=\mu_{1} \otimes \cdots \otimes \mu_{d}$, and $g$ is a $d$-dimensional function $\Delta \subseteq \mathbb{R}^{d} \rightarrow \mathbb{R}$ such that $g(\mathbf{X}) \in L^{2}(\mu)$. Then, we have the Sobol-Hoeffding decomposition [24, 6, 10]:

$$
\begin{aligned}
g(\mathbf{X}) & =g_{0}+\sum_{i=1}^{d} g_{i}\left(X_{i}\right)+\sum_{1 \leq i<j \leq d} g_{i, j}\left(X_{i}, X_{j}\right)+\cdots+g_{1, \ldots, d}\left(X_{1}, \ldots, X_{d}\right) \\
& =\sum_{I \subseteq\{1, \ldots, d\}} g_{I}\left(\mathbf{X}_{I}\right)
\end{aligned}
$$

where the summands are centered and orthogonal. The $g_{i}$ 's are called main effects, and describe the parts of $g$ that are influenced by only one variable. The terms of higher order $g_{i, j}$ 's, $g_{i, j, k}$ 's, etc., are called interactions and involve several variables at a time. The $g_{i, j}$ 's are the second-order interactions, $g_{i, j, k}$ 's the third-order interactions and so on.

From orthogonality in Equation (1), the variance is decomposed:

$$
\operatorname{var}(g(\mathbf{X}))=\sum_{I \subseteq\{1, \ldots, d\}} \operatorname{var}\left(g_{I}\left(\mathbf{X}_{I}\right)\right)
$$

The overall variance is often denoted by $D=\operatorname{var}(g(\mathbf{X}))$, and the variance terms $D_{I}=\operatorname{var}\left(g_{I}\left(\mathbf{X}_{I}\right)\right)$ are called partial variances. The Sobol sensitivity indices $S_{I}$ are then defined as variance ratios [24]:

$$
S_{I}=\frac{D_{I}}{D}
$$

Another global sensitivity measure of interest is the total partial variance, and its normalized version, the total sensitivity index [11] defined by 
considering the supersets of sets of size 1:

$$
D_{i}^{\mathrm{T}}=\sum_{J \supseteq\{i\}} D_{J}, \quad S_{i}^{\mathrm{T}}=\frac{D_{i}^{\mathrm{T}}}{D} .
$$

The total indices allow to detect unessential variables: If $D_{i}^{\mathrm{T}}=0$ then $g(\mathbf{x})$ does not depend on $x_{i}$. An extension to general supersets was proposed by $[12,17]$, who defined the superset importance:

$$
D_{I}^{\text {super }}=\sum_{J \supseteq I} D_{J}, \quad S_{I}^{\text {super }}=\frac{D_{I}^{\text {super }}}{D},
$$

where $I \subseteq\{1, \ldots, d\}$. In the case of a pair of variables $\left\{X_{i}, X_{j}\right\}$, we have: $D_{i, j}^{\text {super }}=\sum_{I \supseteq\{i, j\}} D_{I}$.

\section{2. $D G S M$ and crossed DGSM}

Motivated by the reduction of computational cost, [15] proposed to use the derivative-based global sensitivity measures (DGSM):

$$
\nu_{i}=\int\left(\frac{\partial g(\mathbf{x})}{\partial x_{i}}\right)^{2} d \mu(\mathbf{x}) .
$$

In the equation above it is assumed that $g$ admits a weak directional derivative (i.e. in the sense of distributions) with respect to $x_{i}$ such that $\frac{\partial g(\mathbf{X})}{\partial x_{i}} \in$ $L^{2}(\mu)$. The DGSM play a similar role to total indices in detecting unessential variables: If $\nu_{i}=0$ then $g(\mathbf{x})$ does not depend on $x_{i}$.

Investigating interactions, [7] used the integral of squared crossed derivatives. For second-order interactions, they introduced (assuming similar conditions on the higher-order derivatives of $g$ ):

$$
\nu_{i, j}=\int\left(\frac{\partial^{2} g(\mathbf{x})}{\partial x_{i} \partial x_{j}}\right)^{2} d \mu(\mathbf{x})
$$

and more generally, for $I \subseteq\{1, \ldots, d\}$ :

$$
\nu_{I}=\int\left(\frac{\partial^{|I|} g(\mathbf{x})}{\partial \mathbf{x}_{I}}\right)^{2} d \mu(\mathbf{x}),
$$

with $\partial \mathbf{x}_{I}=\prod_{i \in I} \partial x_{i}$, and $|I|$ is the size of $I$. By analogy to DGSM, when $|I| \geq 2$ we propose to call the quantities $\nu_{I}$ crossed DGSM. 


\subsection{Utilization of superset importance and crossed DGSM}

Superset importance and crossed DGSM are both useful in order to investigate interactions, and discover additive structures in $g$. In practice, the most useful case is for a pair of indices $\{i, j\}$. If either $\nu_{i, j}=0$ or $D_{i, j}^{\text {super }}=0$, then $g$ can be written as a sum of two functions, one that does not depend on $x_{i}$ and the other that does not depend on $x_{j}[12,7]$ :

$$
g(\mathbf{x})=g_{-i}\left(\mathbf{x}_{-i}\right)+g_{-j}\left(\mathbf{x}_{-j}\right)
$$

Equivalently, this means that $X_{i}$ and $X_{j}$ do not interact together, nor together with other variables: $\forall I \supseteq\{i, j\}: D_{I}=0$. In particular - and this is weaker in general - it implies that $D_{i, j}=0$.

\subsection{First-order analysis, second-order analysis. FANOVA graph.}

In applications it is reasonable to perform sequentially so-called firstorder and second-order analysis. The first-order analysis considers single inputs and aims at detecting the non-influential ones, i.e. the $X_{i}$ 's for which

$S_{i}^{T}=0$. First-order sensitivity indices are computed, as well as total sensitivity indices or DGSMs. The second-order analysis considers pairs of inputs, and aims at detecting the non-influential interactions, i.e. the pairs $\{i, j\}$ for which $S_{i, j}^{\text {super }}=0$. This can be used to detect additive structures (see the previous section). Superset importance or crossed DGSMs are computed.

The second-order analysis may generate a large amount of information corresponding to the number of input pairs $p(p-1) / 2$, where $p$ is the problem dimension. Actually $p$ can be lowered to the number of influential variables. Indeed, if $X_{i}$ is non-influential, it does not contribute to the output, neither individually nor in interaction with another input, and thus $S_{i, j}^{\text {super }}=\nu_{i, j}=0$ for all $j$. Such information is conveniently visualized by the way of the so-called FANOVA graph [19], where vertices represent inputs, and edges indicate the presence of interactions involving two inputs simultaneously. The edges widths can then be chosen proportionally to the quantity of interest.

\section{A link between superset importance and crossed DGSM}

In what follows, we consider a class of distributions that satisfy a Poincaré inequality:

$$
\int g(\mathbf{x})^{2} d \mu(\mathbf{x}) \leq C(\mu) \int\|\nabla g(\mathbf{x})\|^{2} d \mu(\mathbf{x})
$$


for all functions $g$ in $L^{2}(\mu)$ such that $\int g(\mathbf{x}) d \mu(\mathbf{x})=0$, and $\|\nabla g\| \in L^{2}(\mu)$. In this paper, a constant $C(\mu)$ satisfying (7) is referred to as a Poincaré constant of $\mu$, and the best possible constant for a given $\mu$ will be called the optimal Poincaré constant, and denoted $C_{\mathrm{opt}}(\mu)$. In that case, i.e. when $C(\mu)=C_{\text {opt }}(\mu)$, it may (but not always) happen that there exists some function $g_{\mathrm{opt}}$ for which Inequality $(7)$ is an equality: Then we say that $g_{\mathrm{opt}}$ is saturating the Poincaré inequality. This is the case for instance for the uniform, exponential and Gaussian distributions (see e.g. [1]). Poincaré inequalities (7) are linked to isoperimetric inequalities in measure theory, and the Poincaré constants are expressed as a function of so-called Cheeger constants [3].

A connection between total indices and DGSM has been established by [27] for the uniform and normal distributions and [16] for general continuous distributions. It states that, under mild conditions:

$$
D_{i}^{\mathrm{T}} \leq C\left(\mu_{i}\right) \nu_{i}
$$

Our main result is that such an inequality can be extended to superset importance and crossed DGSM. For the sake of simplicity, we present it for a pair of variables, but it is true in general (see Remark 1).

Theorem 1. Let us consider $n$ distributions $\mu_{1}, \ldots, \mu_{n}$ on the real line $\mathbb{R}$, and $\mu=\mu_{1} \otimes \cdots \otimes \mu_{n}$. Assume that all $\mu_{i}(i=1, \ldots, n)$ satisfy the Poincaré inequality (7). Let $g: \mathbb{R}^{n} \rightarrow \mathbb{R}$ be a function in $L^{2}(\mu)$, such that all firstorder and crossed second-order partial derivatives are in $L^{2}(\mu)$. Then for all pairs $\{i, j\}(1 \leq i, j \leq n)$,

$$
D_{i, j} \leq D_{i, j}^{\text {super }} \leq C\left(\mu_{i}\right) C\left(\mu_{j}\right) \nu_{i, j}
$$

Furthermore, $C_{\text {opt }}\left(\mu_{i}\right) C_{\text {opt }}\left(\mu_{j}\right)$ is the best constant: If equalities can be achieved in the Poincaré inequalities satisfied by each $\mu_{i}$, then we can have equality in (9).

Proof. The first inequality is straightforward and true for all $g$ in $L^{2}(\mu)$. For the second one, consider the Sobol-Hoeffding decomposition (1) of $g$ and denote $g_{i, j}^{\text {super }}(\mathbf{x}):=\sum_{J \supseteq\{i, j\}} g_{J}\left(\mathbf{x}_{J}\right)$. Since the $g_{J}$ 's are centered and orthogonal, we have:

$$
D_{i, j}^{\text {super }}=\operatorname{var}\left(g_{i, j}^{\text {super }}(\mathbf{x})\right)=\int\left(g_{i, j}^{\text {super }}(\mathbf{x})\right)^{2} d \mu(\mathbf{x}) .
$$


Furthermore we have: $\frac{\partial^{2} g(\mathbf{x})}{\partial x_{i} \partial x_{j}}=\frac{\partial^{2} g_{i j}^{\text {super }}(\mathbf{x})}{\partial x_{i} \partial x_{j}}$, since all terms in (1) that do not contain simultaneously $x_{i}$ and $x_{j}$ vanish by cross derivation. This leads to:

$$
\nu_{i, j}=\int\left(\frac{\partial^{2} g_{i, j}^{\text {super }}(\mathbf{x})}{\partial x_{i} \partial x_{j}}\right)^{2} d \mu(\mathbf{x})
$$

The result then follows from a sequential application of 1-dimensional Poincaré inequalities. Indeed, let us first fix all variables except $x_{i}$. Then:

$$
\int\left(g_{i, j}^{\text {super }}(\mathbf{x})\right)^{2} d \mu_{i}\left(x_{i}\right) \leq C\left(\mu_{i}\right) \int\left(\frac{\partial g_{i, j}^{\text {super }}(\mathbf{x})}{\partial x_{i}}\right)^{2} d \mu_{i}\left(x_{i}\right) .
$$

Similarly, fixing all variables except $x_{j}$ and considering $\frac{\partial g_{i, j}^{\text {super }}(\mathbf{x})}{\partial x_{i}}$, we have:

$$
\int\left(\frac{\partial g_{i, j}^{\text {super }}(\mathbf{x})}{\partial x_{i}}\right)^{2} d \mu_{j}\left(x_{j}\right) \leq C\left(\mu_{j}\right) \int\left(\frac{\partial}{\partial x_{j}} \frac{\partial g_{i, j}^{\text {super }}(\mathbf{x})}{\partial x_{i}}\right)^{2} d \mu_{j}\left(x_{j}\right) .
$$

Then, integrating and combining the two inequalities above gives, together with (10) and (11), the announced inequality.

Finally, to see that $C_{\text {opt }}\left(\mu_{i}\right) C_{\text {opt }}\left(\mu_{j}\right)$ is the best constant, suppose that for each $\mu_{k},(7)$ is saturated with a centered function $g_{\mathrm{opt}}^{k}$ :

$$
\int g_{\mathrm{opt}}^{k}(t)^{2} d \mu_{k}(t)=C_{\mathrm{opt}}\left(\mu_{k}\right) \int\left[\left(g_{\mathrm{opt}}^{k}\right)^{\prime}(t)\right]^{2} d \mu_{k}(t)
$$

Then a direct computation shows that we have equality in (9) with $g\left(x_{i}, x_{j}\right)=$ $g_{\text {opt }}^{i}\left(x_{i}\right) g_{\text {opt }}^{j}\left(x_{j}\right)$ :

$$
D_{i, j}=D_{i, j}^{\text {super }}=C_{\text {opt }}\left(\mu_{i}\right) C_{\text {opt }}\left(\mu_{j}\right) \nu_{i, j}
$$

Remark also that $g(\mathbf{x})=\sum_{k, \ell} g_{\mathrm{opt}}^{k}\left(x_{k}\right) g_{\mathrm{opt}}^{\ell}\left(x_{\ell}\right)$ achieves equality in (9) simultaneously for all pairs $\{i, j\}$.

Remark 1. A similar proof can be used to show that for a general subset $I$, we have: $D_{I}^{\text {super }} \leq \prod_{i \in I} C\left(\mu_{i}\right) \nu_{I}$.

\section{Computation of Poincaré constants on the real line}

In Theorem 1, we can see that Inequality (9) involves only Poincaré constants on the real line (namely the $C\left(\mu_{i}\right)^{\prime}$ 's). There exists an abundant literature on that topic, and some pointers are given in [1]. Here, following [16], 
we assume that $\mu$ is continuous (absolutely continuous with respect to the Lebesgue measure) and focus on some practical results to compute Poincaré constants. We denote by $f$ the probability density function, $F$ its cumulative density function, and $q$ the quantile function. Finally $\widetilde{m}=q(0.5)$ is the median.

First of all, there are at least two cases where optimal constants are known: The uniform and normal distributions (see e.g. [27] or [1]). The constants are given in Table 1.

\begin{tabular}{l|c|l}
\hline Distribution & Optimal Poincaré constant & A case of equality \\
\hline Uniform $\mathcal{U}[a, b]$ & $(b-a)^{2} / \pi^{2}$ & $g(x)=\cos \left(\frac{\pi(x-a)}{b-a}\right)$ \\
Normal $\mathcal{N}\left(\mu, \sigma^{2}\right)$ & $\sigma^{2}$ & $g(x)=x-\mu$ \\
\hline
\end{tabular}

Table 1: Optimal Poincaré constants for the uniform and normal distributions.

In general, however, optimal constants are not available. Fortunately useful Poincaré constants can be derived for some classes of distributions, including in particular log-concave distributions [20].

Definition 1. (log-concave distribution) A continuous distribution $\mu$ is called log-concave if $\log (f)$ is concave.

Three main results are summarized in Table 2. The first one is common to all continuous distributions, and can be found in $[4,3]$. It gives a Poincaré constant that can be computed numerically by maximizing a 1-dimensional function. For log-concave distributions, this maximum has an analytical expression [3], Section 4, and several examples are given in [16] including exponential, Gumbel and Weibull distributions. The last result - useful in applications - is derived for truncated distributions (for a proof see Appendix A).

Remark 2. (Isoperimetric, Cheeger and Poincaré constants). Due to the interpretation of Poincaré inequalities as isoperimetric inequalities [3], there are several constants that are closely linked to each other. The quantity $I s(\mu):=\inf _{x \in \mathbb{R}} \frac{f(x)}{\min (F(x), 1-F(x))}$ is called isoperimetric constant [4]. Its inverse $1 / I s(\mu)$ is often called Cheeger constant [16]. Then $C(\mu)=4 / I s(\mu)^{2}$ is a Poincaré constant for the distribution $\mu$ (see [3]), as reported in Table 2. 


\begin{tabular}{l|c}
\hline Properties of $\mu$ & A Poincaré constant $C(\mu)$ \\
\hline Continuous & $4\left[\sup _{x \in \mathbb{R}} \frac{\min (F(x), 1-F(x))}{f(x)}\right]^{2}$ \\
log-concave & $1 / f(\widetilde{m})^{2}$ \\
log-concave, truncated on $[a, b]$ & $(F(b)-F(a))^{2} / f\left(q\left(\frac{F(a)+F(b)}{2}\right)\right)^{2}$ \\
\hline
\end{tabular}

Table 2: Example of (non-optimal) Poincaré constants for some classes of continuous distributions.

Remark 3. (Boltzmann distributions and erratum in [16]). Continuous distributions $d \mu(x)=f(x) d x$ are sometimes expressed as Boltzmann distributions: $f(x)=c \exp [-v(x)] 1_{\Delta}(x)$, where $c$ is a (non-unique) normalizing constant and $\Delta=\{x \in \mathbb{R}, f(x) \neq 0\}$. If $f$ is log-concave, then the Poincaré constant of Table 2 is equal to $1 / f(\widetilde{m})^{2}=\exp [2 v(\widetilde{m})] / c^{2}$, and the corresponding Cheeger constant (as defined in Remark 2) to $\exp [v(\widetilde{m})] /(2 c)$. The normalizing constant $c$ is sometimes missing in the text of [16]: In Theorem 3.2. (c was omitted in the denominator) and in Table 2, first line: One should read $\sigma / 2 \times \sqrt{2 \pi}$.

\section{Examples for usual analytical functions}

\subsection{Second order polynomials}

Consider a second-order polynomial given by its ANOVA decomposition:

$$
g(\mathbf{X})=\beta_{0}+\sum_{i=1}^{d} \beta_{i}\left(X_{i}-m_{i}\right)+\sum_{1 \leq i<j \leq d} \beta_{i, j}\left(X_{i}-m_{i}\right)\left(X_{j}-m_{j}\right)
$$

where $m_{i}=E\left(X_{i}\right), 1 \leq i \leq d$. Then we have immediately:

$$
D_{i, j}^{\text {super }}=D_{i, j}=\left(\beta_{i, j}\right)^{2} \operatorname{var}\left(X_{i}\right) \operatorname{var}\left(X_{j}\right), \quad \nu_{i, j}=\left(\beta_{i, j}\right)^{2}
$$

Thus, contrarily to superset importance, the crossed DGSM do not depend on the distribution of the $X_{k}$ 's but only on the coefficients of the second-order terms. Consequently, they can give only a rough indication of the interactions importance, but that indication is sufficient to detect the unessential ones: $\nu_{i, j}=0 \Leftrightarrow D_{i, j}=0$ (assuming that $\operatorname{var}\left(X_{i}\right)>0, \operatorname{var}\left(X_{j}\right)>0$ ). 


\subsection{Functions with separated variables}

Following [27], let us consider

$$
g(\mathbf{X})=\prod_{i=1}^{d} \varphi_{i}\left(X_{i}\right)
$$

where $\varphi_{i}\left(X_{i}\right)$ and $\varphi_{i}^{\prime}\left(X_{i}\right)$ are in $L^{2}\left(\mu_{i}\right)$. By denoting $A_{i}=\int \varphi_{i}(t) d \mu_{i}(t)$ and introducing the centered function $\varphi_{i, 0}()=.\varphi_{i}()-.A_{i}$, this is rewritten as:

$$
g(X)=\prod_{i=1}^{d}\left(A_{i}+\varphi_{i, 0}\left(X_{i}\right)\right)
$$

and the ANOVA decomposition of $g$ is obtained by expanding the product (see [25]) and $g_{I}\left(\mathbf{X}_{I}\right)=\prod_{j \notin I} A_{j} \prod_{i \in I} \varphi_{i, 0}\left(X_{i}\right)$. Then a direct computation shows that:

$$
D_{i, j}^{\text {super }}=\prod_{k \notin\{i, j\}}\left(A_{k}^{2}+B_{k}\right) B_{i} B_{j} \quad \nu_{i, j}=\prod_{k \notin\{i, j\}}\left(A_{k}^{2}+B_{k}\right) B_{i}^{\prime} B_{j}^{\prime}
$$

with $B_{i}:=\int \varphi_{i, 0}^{2}(t) d \mu_{i}(t)$ and $B_{i}^{\prime}:=\int\left[\varphi_{i, 0}^{\prime}(t)\right]^{2} d \mu_{i}(t)$. In particular for a non-zero interaction, we have:

$$
\frac{\nu_{i, j}}{D_{i, j}^{\text {super }}}=\frac{B_{i}^{\prime}}{B_{i}} \frac{B_{j}^{\prime}}{B_{j}}
$$

This generalizes the formula given in [27] for first-order indices:

$$
\frac{\nu_{i}}{D_{i}^{\mathrm{T}}}=\frac{B_{i}^{\prime}}{B_{i}}
$$

Their examples with uniform distributions on $[0,1]$ are also immediately extended:

- For the g-function $g(\mathbf{x})=\prod_{i=1}^{d}\left(\left|4 x_{i}-2\right|+a_{i}\right) /\left(1+a_{i}\right)$, the ratio is constant: $\frac{\nu_{i, j}}{D_{i, j}^{\text {super }}}=48^{2}$, and much larger than $\pi^{2}$, the bound given by the optimal Poincaré constant.

- Choosing $\varphi_{i}(t)=t^{m}, \varphi_{j}(t)=t^{n}$ leads to $\frac{\nu_{i, j}}{D_{i, j}^{\text {suer }}} \approx(m+1)^{2}(n+1)^{2}$ for large $m$ and $n$, and the ratio can be arbitrary large. 


\section{Numerical examples and applications}

The aim of this section is to illustrate how DGSM and crossed DGSM can be used in practice. More precisely, the analysis should be based on their upper bounds, denoted by $U_{i}$ and $U_{i, j}$, obtained with Inequalities (8) and (9):

$$
\begin{aligned}
U_{i} & :=C\left(\mu_{i}\right) \frac{\nu_{i}}{D} \geq S_{i}^{T} \\
U_{i, j} & :=C\left(\mu_{i}\right) C\left(\mu_{j}\right) \frac{\nu_{i, j}}{D} \quad \geq S_{i, j}^{\text {super }}
\end{aligned}
$$

In the following, we consider analytical examples and a case study. We perform sequentially first-order analysis in order to detect influential inputs, and a second-order analysis in order to study interactions and discover additive structures, as detailed in Section 2.4. These two analyses imply the estimation of various sensitivity indices. The estimation of variance-based sensitivity indices has been intensively studied since Sobol' formula for closed indices [24]. For total indices and superset importance, two useful formulas can be used, due to [14] and [17]:

$$
\begin{gathered}
D_{i}^{T}=\frac{1}{2} \int\left[f(\mathbf{x})-f\left(z_{i}, \mathbf{x}_{-\mathbf{i}}\right)\right]^{2} d \mu(\mathbf{x}) d \mu_{i}\left(z_{i}\right) \\
D_{i, j}^{\text {super }}=\frac{1}{4} \int\left[f(\mathbf{x})-f\left(x_{i}, z_{j}, \mathbf{x}_{-\mathbf{i}, \mathbf{j}}\right)\right. \\
\left.-f\left(z_{i}, x_{j}, \mathbf{x}_{-\mathbf{i}, \mathbf{j}}\right)+f\left(z_{i}, z_{j}, \mathbf{x}_{-\mathbf{i}, \mathbf{j}}\right)\right]^{2} d \mu(\mathbf{x}) d \mu_{i}\left(z_{i}\right) d \mu_{j}\left(z_{j}\right)
\end{gathered}
$$

Accurate estimators are derived by using Monte Carlo estimates of the integrals above. They share good properties, studied in [8]: They are positive, unbiased and asymptotically efficient. Furthermore, they are identically zero if the "true" index is zero.

For DGSM and crossed DGSM, estimations are obtained by using also Monte Carlo estimates of the integrals in Equations (5) and (6). In addition, the derivatives should be replaced by finite differences when the gradient and/or Hessian are not supplied. In this section, we use finite differences. In both cases, it is easy to see that the estimators share the same property as above: They are identically zero if the true index is zero.

Finally, notice that all the integrals could be replaced by Quasi-Monte Carlo estimates rather than Monte Carlo ones, but this is beyond the scope of the present paper. 
Remark 4. Formula (16) contains a crossed finite difference inside the square. It is thus very similar to the crossed DGSM definition (6). This can be used to prove Inequality (9) from (16), as detailed in Appendix B.

\subsection{Analytical examples}

\subsubsection{Ishigami function}

The Ishigami function is a popular toy example in sensitivity analysis, due to the presence of non-linearities and a strong (non-linear) interaction. It is defined on $\Delta=[-\pi, \pi]^{3}$ by:

$$
f\left(x_{1}, x_{2}, x_{3}\right)=\sin \left(x_{1}\right)+7 \sin \left(x_{2}\right)^{2}+0.1 x_{3}^{4} \sin \left(x_{1}\right) .
$$

We consider $f\left(X_{1}, X_{2}, X_{3}\right)$, assuming that $X_{1}, X_{2}, X_{3}$ are independent random variables from the uniform distribution on $[-\pi, \pi]$. Each index is estimated a 100 times on different Monte Carlo samples of size 1000. The resulting mean and standard deviation values of the sensitivity analysis are reported in Tables 3 and 4 . The theoretical values, here calculable, are also indicated. We observe that the upper bounds are quite large, though the optimal Poincaré constant was used. No major conclusion is obtained from Table 3, since all inputs are influential. We see, however, that the ranking of inputs is different whether it is based on $\hat{S}_{i}^{T}$ or $\hat{U}_{i}$. The second-order analysis (Table 4) shows that the non-influential interactions $\{2,1\},\{2,3\}$ are correctly detected by the $\hat{U}_{i, j}$ 's. Notice that the estimation is exactly zero when the index is zero, a property of the Liu and Owen's estimator (see above). This implies that there are no interactions (at any order) involving both $X_{2}$ and $X_{1}$, and no interactions (at any order) involving both $X_{3}$ and $X_{1}$, revealing an additive structure for $f$ :

$$
f\left(x_{1}, x_{2}, x_{3}\right)=f_{1,3}\left(x_{1}, x_{3}\right)+f_{2}\left(x_{2}\right)
$$

\begin{tabular}{|l|r|lll|lll|}
\hline Input & $S_{i}$ & $S_{i}^{T}$ & $\widehat{m}\left(\hat{S}_{i}^{T}\right)$ & $\widehat{s d}\left(\hat{S}_{i}^{T}\right)$ & $U_{i}$ & $\widehat{m}\left(\hat{U}_{i}\right)$ & $\widehat{s d}\left(\hat{U}_{i}\right)$ \\
\hline$X_{1}$ & 0.314 & 0.558 & 0.558 & $(0.047)$ & 2.230 & 2.234 & $(0.146)$ \\
$X_{2}$ & 0.442 & 0.442 & 0.442 & $(0.015)$ & 7.079 & 7.048 & $(0.163)$ \\
$X_{3}$ & 0 & 0.244 & 0.243 & $(0.016)$ & 3.174 & 3.213 & $(0.221)$ \\
\hline
\end{tabular}

Table 3: First-order analysis for the Ishigami function (uniform inputs). Upper bounds are computed with $C_{\text {opt }}(\mu)=4$. Standard deviations are obtained with 100 replicates. 


\begin{tabular}{|l|r|lll|lll|}
\hline Input pair & $S_{i, j}$ & $S_{i, j}^{\text {super }}$ & $\widehat{m}\left(\hat{S}_{i, j}^{\text {super }}\right)$ & $\widehat{s d}\left(\hat{S}_{i, j}^{\text {super }}\right)$ & $U_{i, j}$ & $\widehat{m}\left(\hat{U}_{i, j}\right)$ & $\widehat{s d}\left(\hat{U}_{i, j}\right)$ \\
\hline$X_{1}: X_{2}$ & 0 & 0 & 0 & $(0)$ & 0 & 0 & $(0)$ \\
$X_{1}: X_{3}$ & 0.244 & 0.244 & 0.241 & $(0.020)$ & 12.698 & 12.686 & $(0.828)$ \\
$X_{2}: X_{3}$ & 0 & 0 & 0 & $(0)$ & 0 & 0 & $(0)$ \\
\hline
\end{tabular}

Table 4: Second-order analysis for the Ishigami function (uniform inputs). Upper bounds are computed with $C_{\text {opt }}(\mu)=4$. Standard deviations are obtained with 100 replicates.

\subsubsection{A 6-dimensional block-additive function}

Following [19], we consider the 6-dimensional function in $L^{2}$ :

$$
a\left(X_{1}, \ldots, X_{6}\right)=\cos \left(\left[1, X_{1}, X_{5}, X_{3}\right] \phi\right)+\sin \left(\left[1, X_{4}, X_{2}, X_{6}\right] \gamma\right)
$$

with $\phi=[-0.8,-1.1,1.1,1]^{T}, \gamma=[0.5,0.9,1,-1.1]^{T}$, and where $X_{1}, \ldots, X_{6}$ are assumed independent and uniformly distributed on $\Delta=[-1,1]^{6}$. The results for sensitivity analysis are reported in Tables 5 and 6 . Again each estimation is performed with a Monte Carlo sample of size 1000 and repeated 100 times. Table 5 shows that all variables are influential, either by looking at $S_{i}^{T}$ or $U_{i}$. Looking at interactions between pairs of inputs, Table 6 clearly detects inactive interactions, either by looking at $\hat{S}_{i, j}^{\text {super }}$ or $\hat{U}_{i, j}$. Again, the estimation is exactly zero when the index is zero. The corresponding FANOVA graphs (see 2.4) are represented in Figure 1, which are very similar here. Two groups are visible: $\{1,3,5\}$ and $\{2,4,6\}$, meaning that there are no interactions (at any order) between $X_{i}$ and $X_{j}$ when $i$ belongs to the first group, and $j$ to the second one. Thus the Sobol-Hoeffding decomposition (1) implies that the 'a' function has the block-additive form:

$$
a\left(X_{1}, \ldots, X_{6}\right)=a_{1,3,5}\left(X_{1}, X_{3}, X_{5}\right)+a_{2,4,6}\left(X_{2}, X_{4}, X_{6}\right)
$$

which is the correct structure of the 'a' function.

\subsection{A case study}

We consider the flood model presented in [16]. The output is the maximal annual overflow (in meters), denoted by $S$ :

$$
S=Z_{v}+H-H_{d}-C_{b} \quad \text { with } \quad H=\left(\frac{Q}{B K_{s} \sqrt{\frac{Z_{m}-Z_{v}}{L}}}\right)^{0.6}
$$




\begin{tabular}{|l|r|lll|lll|}
\hline Input & $S_{i}$ & $S_{i}^{T}$ & $\widehat{m}\left(\hat{S}_{i}^{T}\right)$ & $\widehat{s d}\left(\hat{S}_{i}^{T}\right)$ & $U_{i}$ & $\widehat{m}\left(\hat{U}_{i}\right)$ & $\widehat{s d}\left(\hat{U}_{i}\right)$ \\
\hline$X_{1}$ & 0.110 & 0.231 & 0.231 & $(0.012)$ & 0.329 & 0.329 & $(0.007)$ \\
$X_{2}$ & 0.143 & 0.214 & 0.215 & $(0.009)$ & 0.285 & 0.285 & $(0.005)$ \\
$X_{3}$ & 0.086 & 0.196 & 0.197 & $(0.010)$ & 0.272 & 0.272 & $(0.006)$ \\
$X_{4}$ & 0.112 & 0.176 & 0.176 & $(0.008)$ & 0.231 & 0.231 & $(0.004)$ \\
$X_{5}$ & 0.110 & 0.231 & 0.232 & $(0.011)$ & 0.329 & 0.329 & $(0.007)$ \\
$X_{6}$ & 0.180 & 0.256 & 0.256 & $(0.011)$ & 0.345 & 0.345 & $(0.007)$ \\
\hline
\end{tabular}

Table 5: First-order analysis for the 'a' function (uniform inputs). Upper bounds are computed with $C_{\mathrm{opt}}(\mu)=4 / \pi^{2}$. Standard deviations are obtained with 100 replicates.

\begin{tabular}{|l|r|lll|lll|}
\hline Input pair & $S_{i, j}$ & $S_{i, j}^{\text {super }}$ & $\widehat{m}\left(\hat{S}_{i, j}^{\text {super }}\right)$ & $\widehat{s d}\left(\hat{S}_{i, j}^{\text {super }}\right)$ & $U_{i, j}$ & $\widehat{m}\left(\hat{U}_{i, j}\right)$ & $\widehat{s d}\left(\hat{U}_{i, j}\right)$ \\
\hline$X_{1}: X_{2}$ & 0 & 0 & 0 & $(0)$ & 0 & 0 & $(0)$ \\
$X_{1}: X_{3}$ & 0.043 & 0.067 & 0.067 & $(0.005)$ & 0.133 & 0.132 & $(0.003)$ \\
$X_{1}: X_{4}$ & 0 & 0 & 0 & $(0)$ & 0 & 0 & $(0)$ \\
$X_{1}: X_{5}$ & 0.055 & 0.078 & 0.080 & $(0.006)$ & 0.161 & 0.160 & $(0.004)$ \\
$X_{1}: X_{6}$ & 0 & 0 & 0 & $(0)$ & 0 & 0 & $(0)$ \\
$X_{2}: X_{3}$ & 0 & 0 & 0 & $(0)$ & 0 & 0 & $(0)$ \\
$X_{2}: X_{4}$ & 0.018 & 0.040 & 0.039 & $(0.004)$ & 0.085 & 0.085 & $(0.002)$ \\
$X_{2}: X_{5}$ & 0 & 0 & 0 & $(0)$ & 0 & 0 & $(0)$ \\
$X_{2}: X_{6}$ & 0.031 & 0.053 & 0.052 & $(0.005)$ & 0.127 & 0.127 & $(0.003)$ \\
$X_{3}: X_{4}$ & 0 & 0 & 0 & $(0)$ & 0 & 0 & $(0)$ \\
$X_{3}: X_{5}$ & 0.043 & 0.067 & 0.067 & $(0.005)$ & 0.133 & 0.132 & $(0.003)$ \\
$X_{3}: X_{6}$ & 0 & 0 & 0 & $(0)$ & 0 & 0 & $(0)$ \\
$X_{4}: X_{5}$ & 0 & 0 & 0 & $(0)$ & 0 & 0 & $(0)$ \\
$X_{4}: X_{6}$ & 0.024 & 0.046 & 0.045 & $(0.004)$ & 0.103 & 0.103 & $(0.002)$ \\
$X_{5}: X_{6}$ & 0 & 0 & 0 & $(0)$ & 0 & 0 & $(0)$ \\
\hline
\end{tabular}

Table 6: Second-order analysis for the 'a' function (uniform inputs). Upper bounds are computed with $C_{\mathrm{opt}}(\mu)=4 / \pi^{2}$. Standard deviations are obtained with 100 replicates.

where $H$ is the maximal annual height of the river (in meters). The 8 inputs are assumed to be independent random variables, with distributions recalled in Table 7.

The first-order analysis was performed in [16], and 3 nonessential inputs were detected. We base the second-order analysis on the 5 remaining ones: $X_{1}=Q, X_{2}=K_{s}, X_{3}=Z_{v}, X_{5}=H_{d}, X_{6}=C_{b}$. We fix the non-influential inputs at their mean value and estimate the $\hat{S}_{i, j}^{\text {super }}$ and $\hat{U}_{i, j}$ using a Monte 

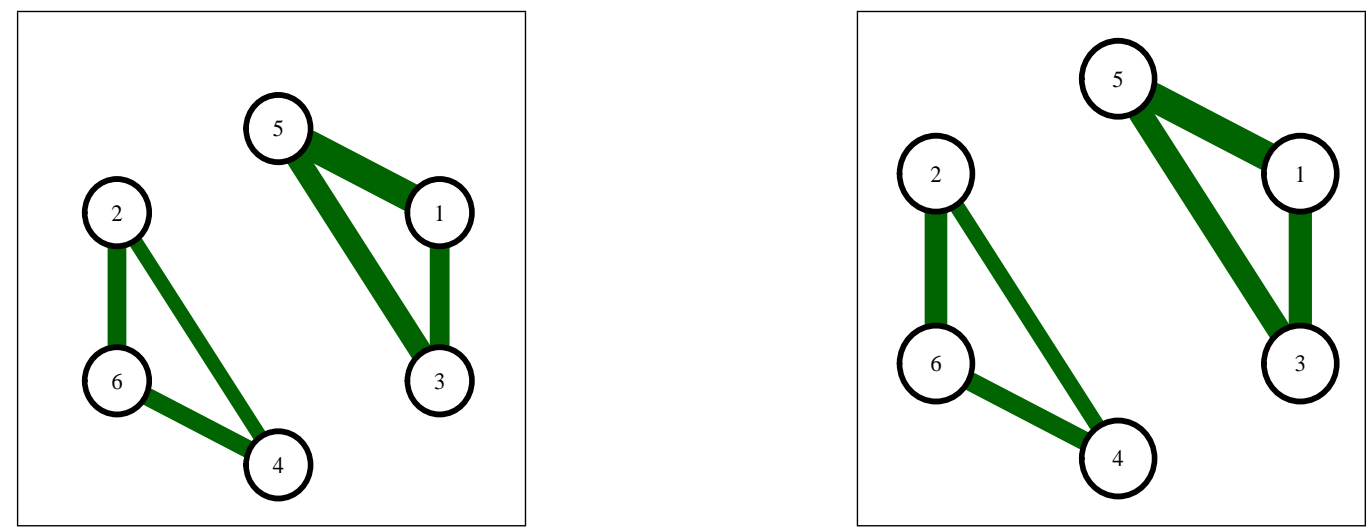

Figure 1: FANOVA graphs of $\hat{S}_{i, j}^{\text {super }}$ (left) and $\hat{U}_{i, j}$ (right) for the 'a' function.

\begin{tabular}{lcrr} 
Input & Description & Unit & Probability distribution \\
\hline$X_{1}=Q$ & Maximal annual flowrate & $\mathrm{m}^{3} / \mathrm{s}$ & $\begin{array}{r}\text { Gumbel } \mathcal{G}(1013,558), \\
\text { truncated on }[500,3000]\end{array}$ \\
$X_{2}=K_{s}$ & Strickler coefficient & - & Normal $\mathcal{N}(30,8)$, \\
& & & truncated on $[15,+\infty[$ \\
$X_{3}=Z_{v}$ & River downstream level & $\mathrm{m}$ & Triangular $\mathcal{T}(49,50,51)$ \\
$X_{4}=Z_{m}$ & River upstream level & $\mathrm{m}$ & Triangular $\mathcal{T}(54,55,56)$ \\
$X_{5}=H_{d}$ & Dyke height & $\mathrm{m}$ & Uniform $\mathcal{U}[7,9]$ \\
$X_{6}=C_{b}$ & Bank level & $\mathrm{m}$ & Triangular $\mathcal{T}(55,55.5,56)$ \\
$X_{7}=L$ & River stretch & $\mathrm{m}$ & $\mathcal{T}(4990,5000,5010)$ \\
$X_{8}=B$ & River width & $\mathrm{m}$ & Triangular $\mathcal{T}(295,300,305)$ \\
\hline
\end{tabular}

Table 7: Input variables of the flood model and their probability distributions.

Carlo sample size of 20,000. The true amount of variance explained by interactions is here very small, around 1\%. The results are reported in Table 8 and visualized on Figure 2. Departures are visible between the results obtained with variance-based sensitivity indices and derivative-based ones. One reason is that the Poincaré constants used are not optimal, and do not have the same order of magnitude. In particular one of them is very large due to the fat tail of the Gumbel distribution. Consequently we see that the upper bounds are rather rough. The second-order analysis based on crossed DGSMs succeeds in detecting the nonessential interactions, after inspecting 
Table 8. However this result must be considered with much care here, since it also corresponds to low values of Poincaré constants products. Similarly we are very lucky here to rank at first place the most influential interaction, corresponding to the highest product $C\left(\mu_{i}\right) C\left(\mu_{j}\right)$.

Finally, we consider the second output, a cost estimation, proposed in [16]. The same 5 inputs are influential and again the interaction indices are estimated on a Monte Carlo size of 20,000.. The true amount of variance explained by the interactions is about $10 \%$. The second-order analysis clearly shows a difference in ranking, as shown in Figure 3. This may be due either to the form of the function considered, or to the heterogeneity of distributions as explained above. Notice however that crossed DGSM conclude correctly to the absence of non-influential interactions.

\begin{tabular}{|c|c|c|r|}
\hline & $\hat{S}_{i, j}^{\text {super }}$ & $\hat{U}_{i, j}$ & $C\left(\mu_{i}\right) C\left(\mu_{j}\right)$ \\
\hline$X_{1}: X_{2}$ & 0.008235 & 1.837809 & 819887392.342 \\
$X_{1}: X_{3}$ & 0.000178 & 0.011691 & 2165585.265 \\
$X_{1}: X_{5}$ & 0.000000 & 0.001947 & 877678.649 \\
$X_{1}: X_{6}$ & 0.000000 & 0.000000 & 541396.316 \\
$X_{2}: X_{3}$ & 0.000070 & 0.003758 & 378.599 \\
$X_{2}: X_{5}$ & 0.000000 & 0.000000 & 153.440 \\
$X_{2}: X_{6}$ & 0.000000 & 0.000000 & 94.650 \\
$X_{3}: X_{5}$ & 0.000000 & 0.000000 & 0.405 \\
$X_{3}: X_{6}$ & 0.000000 & 0.000000 & 0.250 \\
$X_{5}: X_{6}$ & 0.000000 & 0.000000 & 0.101 \\
\hline
\end{tabular}

Table 8: Second-order analysis for the overflow output of the flood model, limited to the influential inputs.

\section{Conclusion}

This paper focuses on the quantification of interactions in global sensitivity analysis. We considered the integral of squared crossed derivatives introduced in statistical learning [7], that we call crossed DGSM by analogy to DGSM [26]. We show that there is a Poincaré-type inequality between superset importance [17] and the crossed DGSM. This extends to interaction the link between total effects and DGSM $[27,16]$. 

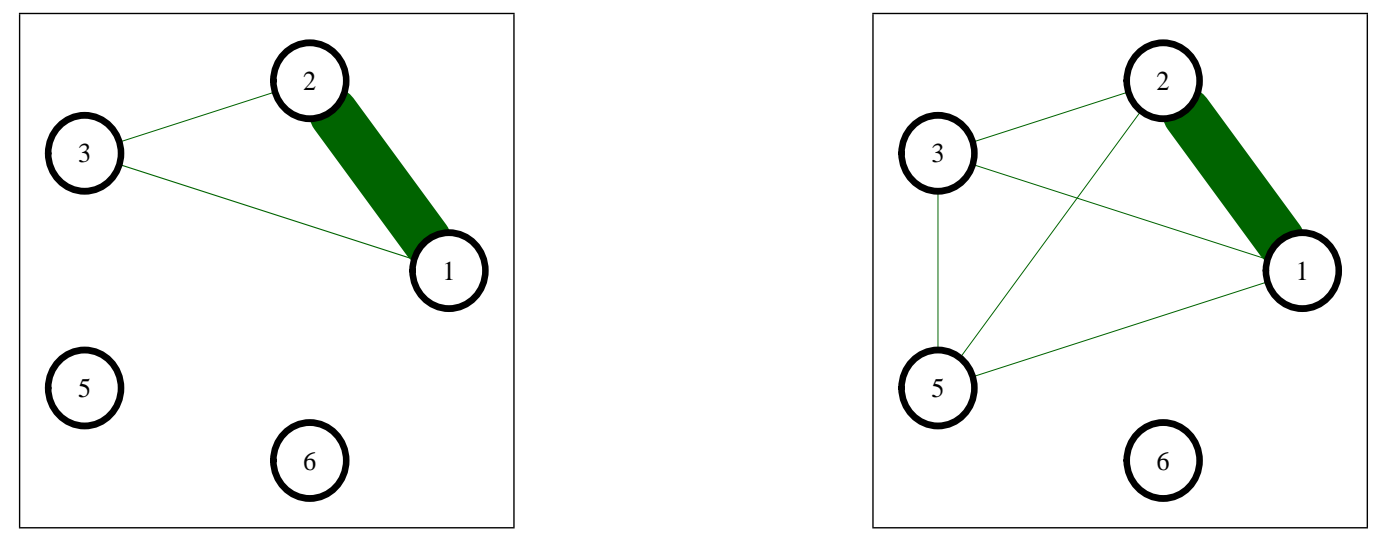

Figure 2: FANOVA graphs of $\hat{S}_{i, j}^{\text {super }}$ (left) and $\hat{U}_{i, j}$ (right) for the overflow output of the flood model, limited to the influential inputs.
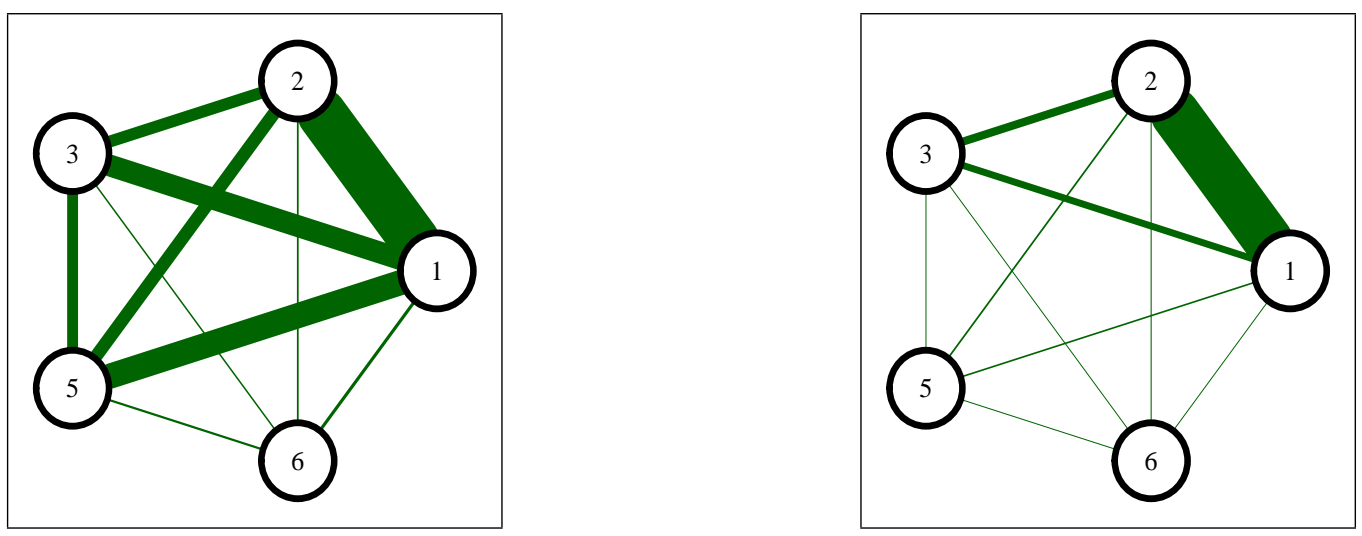

Figure 3: FANOVA graphs of $\hat{S}_{i, j}^{\text {super }}$ (left) and $\hat{U}_{i, j}$ (right) for the cost output of the flood model, limited to the influential inputs.

This new inequality can be used to detect additive structures in black-box functions by detecting pairs of inputs that do not interact together (see $[12,19,8])$. We investigated their practical utilization with two analytical examples involving uniform inputs and one case study with several heterogeneous input distributions. For that purpose, we recalled some facts about Poincaré inequalities, and gave a new expression of a Poincaré constant for truncated log-concave distributions. The crossed DGSM performed well in the examples, for which the optimal Poincaré constant is known. In particular, the additive structure of a 6 -dimensional function was recovered. On the other hand, the case study showed some limitations of the crossed DGSM, 
also shared by the usual DGSM (see [27] for a discussion). In particular, some Poincaré constants used are not optimal and sometimes very large, and the inequality was not informative for several pairs of inputs. In such cases, an idea for future research could be to investigate the use of transformations of the inputs into uniformly or normally distributed ones, which simplifies the input distributions but may introduce undesirable non-linearities in the new function obtained by composition.

\section{Acknowledgements}

Parts of this work have been backed by French National Research Agency (ANR) through COSINUS program (project COSTA BRAVA noANR-09COSI-015), or conducted within the frame of the ReDice Consortium, gathering industrial (CEA, EDF, IFPEN, IRSN, Renault) and academic (Ecole des Mines de Saint-Etienne, INRIA, and the University of Bern) partners around advanced methods for Computer Experiments. The financial support of the DFG (SFB708: Project C3) is also gratefully acknowledged. We thank Fabrice Gamboa and Franck Barthe for helpful discussions.

\section{Appendix A. Poincaré constants for truncated log-concave distri- butions.}

Proposition 1. Let $\mu$ be a log-concave distribution. Let $a, b$ be two real numbers such that $-\infty \leq a<b \leq+\infty$, and consider the truncated distribution $\mu_{a, b}$ defined on $[a, b]$ by its cdf $F_{a, b}(x)=\frac{F(x)-F(a)}{F(b)-F(a)} 1_{[a, b]}(x)$, where $1_{[a, b]}$ denotes the indicator function. Then $\mu_{a, b}$ satisfies a Poincaré inequality with $C\left(\mu_{a, b}\right)=(F(b)-F(a))^{2} / f\left(q\left(\frac{F(a)+F(b)}{2}\right)\right)^{2}$.

Proof. As a truncation of a log-concave distribution, $\mu_{a, b}$ is log-concave (see e.g. [2]), and thus satisfies a Poincaré inequality with $C\left(\mu_{a, b}\right)=1 / f_{a, b}\left(\widetilde{m}_{a, b}\right)^{2}$, where $f_{a, b}$ and $\widetilde{m}_{a, b}$ are respectively the pdf and the median of $\mu_{a, b}$. Now, a direct computation shows that $f_{a, b}(x)=f(x) /(F(b)-F(a)) 1_{[a, b]}(x)$ and $\widetilde{m}_{a, b}=q\left(\frac{F(a)+F(b)}{2}\right)$. 


\section{Appendix B. A second proof of Inequality (9).}

As mentioned in Remark 4, it is possible to derive Inequality (9) from (16). Without loss of generality, we consider the case $i=1, j=2$. The superset importance is expressed as the integral of a squared difference ([17]):

$$
D_{1,2}^{\text {superset }}=\frac{1}{4} \int\left(h\left(x_{1}, x_{2}, z_{1}, z_{2}, \mathbf{z}_{-\{1,2\}}\right)\right)^{2} d \mu\left(x_{1}\right) d \mu\left(x_{2}\right) d \mu(\mathbf{z})
$$

with:

$h\left(x_{1}, x_{2}, \mathbf{z}\right)=g\left(z_{1}, z_{2}, \mathbf{z}_{-\{1,2\}}\right)-g\left(x_{1}, z_{2}, \mathbf{z}_{-\{1,2\}}\right)-g\left(z_{1}, x_{2}, \mathbf{z}_{-\{1,2\}}\right)+g\left(x_{1}, x_{2}, \mathbf{z}_{-\{1,2\}}\right)$

Notice that $h$ is a crossed finite difference:

$$
h\left(x_{1}, x_{2}, \mathbf{z}\right)=g_{2}\left(z_{2} ; x_{1}, \mathbf{z}\right)-g_{2}\left(x_{2} ; x_{1}, \mathbf{z}\right)
$$

with $g_{2}\left(\bullet ; x_{1}, \mathbf{z}\right)=g\left(z_{1}, \bullet, \mathbf{z}_{-\{1,2\}}\right)-g\left(x_{1}, \bullet, \mathbf{z}_{-\{1,2\}}\right)$. Let us first temporarily fix all variables except $x_{2}$ and $z_{2}$. Remark that $k:\left(x_{2}, z_{2}\right) \rightarrow h\left(x_{1}, x_{2}, \mathbf{z}\right)$ is a zero mean function with respect to $\mu_{2} \otimes \mu_{2}$. Now, if $\mu_{i}$ satisfies a Poincaré inequality, then so does $\mu_{i} \otimes \mu_{i}$ and the same Poincaré constant can be used: $C\left(\mu_{i} \otimes \mu_{i}\right)=C\left(\mu_{i}\right),(i=1,2)$, see e.g. [1]. Therefore, applying (7) to $k$ gives:

$$
\begin{aligned}
\int_{\mathbb{R}^{2}} h\left(x_{1}, x_{2}, \mathbf{z}\right)^{2} d \mu_{2}\left(x_{2}\right) d \mu_{2}\left(z_{2}\right) & \leq C\left(\mu_{2}\right) \int_{\mathbb{R}^{2}}\left\|\nabla h\left(x_{1}, x_{2}, \mathbf{z}\right)\right\|^{2} d \mu_{2}\left(x_{2}\right) d \mu_{2}\left(z_{2}\right) \\
& =2 C\left(\mu_{2}\right) \int_{\mathbb{R}}\left(\frac{\partial g_{2}\left(x_{2} ; x_{1}, \mathbf{z}\right)}{\partial x_{2}}\right)^{2} d \mu_{2}\left(x_{2}\right)
\end{aligned}
$$

Similarly, remark that $\frac{\partial g_{2}\left(x_{2} ; x_{1}, \mathbf{z}\right)}{\partial x_{2}}=g_{1}\left(z_{1} ; x_{2}, \mathbf{z}\right)-g_{1}\left(x_{1} ; x_{2}, \mathbf{z}\right)$ with $g_{1}\left(\bullet ; x_{2}, \mathbf{z}\right)=$ $\frac{\partial g\left(\boldsymbol{\bullet}, x_{2}, \mathbf{z}_{-\{1,2\}}\right)}{\partial x_{2}}$. Thus, by considering that all variables are fixed but $x_{1}$ and $z_{1}$, and applying $(7)$ to the zero-mean function $\left(x_{1}, z_{1}\right) \rightarrow \frac{\partial g_{2}\left(x_{2} ; x_{1}, \mathbf{z}\right)}{\partial x_{2}}$, we get:

$$
\int_{\mathbb{R}^{2}}\left(\frac{\partial g_{2}\left(x_{2} ; x_{1}, \mathbf{z}\right)}{\partial x_{2}}\right)^{2} d \mu_{1}\left(x_{1}\right) d \mu_{1}\left(z_{1}\right) \leq 2 C\left(\mu_{1}\right) \int_{\mathbb{R}}\left(\frac{\partial g_{1}\left(x_{1} ; x_{2}, \mathbf{z}\right)}{\partial x_{1}}\right)^{2} d \mu_{1}\left(x_{1}\right)
$$

Then, by combining the two inequalities above and integrating over all variables, we obtain the desired inequality:

$$
D_{1,2}^{\text {superset }} \leq C\left(\mu_{1}\right) C\left(\mu_{2}\right) \int\left(\frac{\partial^{2} g(\mathbf{x})}{\partial x_{1} \partial x_{2}}\right)^{2} d \mu(\mathbf{x})
$$




\section{References}

[1] C. Ané, S. Blachère, D. Chafaï, P. Fougères, I. Gentil, F. Malrieu, C. Roberto, G. Scheffer, Sur les inégalités de Sobolev logarithmiques, volume 10 of Panoramas et Synthèses, Société Mathématique de France, Paris, 2000.

[2] M. Bagnoli, T. Bergstrom, Log-concave probability and its applications, Economic Theory 26 (2005) 445-469.

[3] S.G. Bobkov, Isoperimetric and analytic inequalities for log-concave probability measures, The Annals of Probability 27 (1999) 1903-1921.

[4] S.G. Bobkov, C. Houdré, Isoperimetric constants for product probability measures, The Annals of Probability 25 (1997) 184-205.

[5] D. Cacuci, Sensitivity and uncertainty analysis - Theory, Chapman \& Hall/CRC, Paris, 2003.

[6] B. Efron, C. Stein, The jackknife estimate of variance, The Annals of Statistics 9 (1981) 586-596.

[7] J. Friedman, B. Popescu, Predictive Learning via Rule Ensembles, The Annals of Applied Statistics 2 (2008) 916-954.

[8] J. Fruth, O. Roustant, S. Kuhnt, Total interaction index: A variancebased sensitivity index for interaction screening, Journal of Statistical Planning and Inference 147 (2014) 212-223.

[9] A. Griewank, G. Corliss, Automatic differentiation of algorithms: Theory, implementation and application, SIAM, Philadelphia, 1991.

[10] W. Hoeffding, A class of statistics with asymptotically normal distributions, Annals of Mathematical Statistics 19 (1948) 293-325.

[11] T. Homma, A. Saltelli, Importance measures in global sensitivity analysis of nonlinear models, Reliability Engineering \& System Safety 52 (1996) 1-17.

[12] G. Hooker, Discovering additive structure in black box functions, in: Proceedings of KDD 2004, ACM DL, pp. 575-580. 
[13] B. Iooss, A.L. Popelin, G. Blatman, C. Ciric, F. Gamboa, S. Lacaze, M. Lamboni, Some new insights in derivative-based global sensitivity measures, in: Proceedings of PSAM 11 \& ESREL 2012 Conference, Helsinki, Finland, pp. 1094-1104.

[14] M. Jansen, Analysis of variance designs for model output, Computer Physics Communication 117 (1999) 25-43.

[15] S. Kucherenko, M. Rodriguez-Fernandez, C. Pantelides, N. Shah, Monte carlo evaluation of derivative-based global sensitivity measures, Reliability Engineering and System Safety 94 (2009) 1135-1148.

[16] M. Lamboni, B. Iooss, A.L. Popelin, F. Gamboa, Derivative-based global sensitivity measures: General links with Sobol' indices and numerical tests, Mathematics and Computers in Simulation 87 (2013) 45-54.

[17] R. Liu, A. Owen, Estimating mean dimensionality of analysis of variance decompositions, Journal of the American Statistical Association 101 (2006) 712-721.

[18] A. Marrel, B. Iooss, B. Laurent, O. Roustant, Calculations of the Sobol indices for the Gaussian process metamodel, Reliability Engineering and System Safety 94 (2009) 742-751.

[19] T. Muehlenstaedt, O. Roustant, L. Carraro, S. Kuhnt, Data-driven Kriging models based on FANOVA-decomposition, Statistics \& Computing 22 (2012) 723-738.

[20] A. Prékopa, Logarithmic concave measures with applications to stochastic programming, Acta Scientiarium Mathematicarum 32 (1971) 301316.

[21] M. Rodriguez-Fernandez, J. Banga, F. Doyle, Novel global sensitivity analysis methodology accounting for the crucial role of the distribution of input parameters: application to systems biology models, International Journal of Robust Nonlinear Control 22 (2012) 1082-1102.

[22] A. Saltelli, K. Chan, E. Scott, Sensitivity analysis, Wiley series in probability and statistics, Wiley, Chichester, 2000. 
[23] A. Saltelli, M. Ratto, T. Andres, F. Campolongo, J. Cariboni, D. Gatelli, M. Saisana, S. Tarantola, Global Sensitivity Analysis: The Primer, Wiley-Interscience, 2008.

[24] I. Sobol, Sensitivity estimates for non linear mathematical models, Mathematical Modelling and Computational Experiments 1 (1993) 407414 .

[25] I. Sobol, Theorems and examples on high dimensional model representation, Reliability Engineering and System Safety 79 (2003) 187-193.

[26] I. Sobol, A. Gershman, On an alternative global sensitivity estimator, in: Proceedings of SAMO 1995, Belgirate, Italy, pp. 40-42.

[27] I. Sobol, S. Kucherenko, Derivative-based global sensitivity measures and the link with global sensitivity indices, Mathematics and Computers in Simulation 79 (2009) 3009-3017.

[28] I. Sobol, S. Kucherenko, A new derivative based importance criterion for groups of variables and its link with the global sensitivity indices, Computer Physics Communications 181 (2010) 1212-1217.

[29] C. Storlie, J. Helton, Multiple predictor smoothing methods for sensitivity analysis: Description of techniques, Reliability Engineering and System Safety 93 (2008) 28-54. 